\title{
Effects of non-equally biaxial misfit strains on the phase diagram and dielectric properties of epitaxial ferroelectric thin films
}

\author{
Jie Wang and Tong-Yi Zhang* \\ Department of Mechanical Engineering \\ Hong Kong University of Science and Technology \\ Clear Water Bay, Kowloon, Hong Kong, China
}

\begin{abstract}
The effects of non-equally biaxial in-plane misfit strains on the equilibrium polarization states and the dielectric properties of single-domain epitaxial ferroelectric thin films are investigated by a nonlinear thermodynamic theory. The "misfit strain-misfit strain" and "temperature-misfit strain" phase diagrams for single-domain $\mathrm{BaTiO}_{3}(\mathrm{BT})$ and $\mathrm{PbTiO}_{3}(\mathrm{PT})$ thin films grown on tetragonal substrates are developed by minimizing the Helmholtz free energy. The non-equally biaxial misfit strains cause the presence of two in-plane tetragonal ferreoelectric phases, $a_{1}$ $\left(P_{1} \neq 0, P_{2}=P_{3}=0\right)$ and $a_{2}\left(P_{2} \neq 0, P_{1}=P_{3}=0\right)$, in both BT and PT thin films, which do not exist if the misfit strains are equally biaxial.
\end{abstract}

\footnotetext{
*Corresponding author, Tel: (852) 2358-7192, Fax: (852) 2358-1543, E-mail: mezhangt@ust.hk
} 
Ferroelectric thin films have been found wide applications in new generations of dynamic random access memories, nonvolatile memories, tunable microwave devices, and other microelectronic and electro-optic devices. ${ }^{1-5}$ The misfit strains between a ferroelectric thin film and its substrate are able to change the Curie temperature, the order of the paraelectric/ferroelectric phase transition, the equilibrium polarization states, and the dielectric properties of the material. ${ }^{6}$ Certainly, a deep understanding of the relationship between the misfit strains and the material properties will provide guidelines on how to control the material properties by manipulating the misfit strains, which might be crucial for applications of ferroelectric thin films. Great progress has been achieved in the theoretical study of the misfit strains effect, especially, by using the powerful thermodynamics theories, ${ }^{6-16}$ and the "misfit strain-temperature" phase diagrams have been constructed. ${ }^{6,12-13}$ However, the previous work has investigated only equally biaxial misfit strains, called the isotropic misfit strains also in the literature, which are induced when ferroelectric thin films are grown on cubic substrates. If ferroelectric thin films are grown on tetragonal substrates, the misfit strain along a crystalline axis will differ from the misfit strain along the other crystalline axis, which are called nonequally biaxial (or anisotropic) misfit strains, as illustrated by recent experimental studies. ${ }^{17-19}$ The non-equally biaxial misfit strains may induce new material properties and thus open up other possibilities for the design of electronic devices.

In the present work, we study a single-domain ferroelectric thin film epitaxially grown on a thick tetragonal substrate and assume the film/substrate interface is coherent. Using the Voigt matrix notation of strains and the rectangular Cartesian coordinator system with the $x_{3}$ axis perpendicular to the film/substrate interface, we may define the non-equally biaxial in-plane 
misfit strains as $e_{1}=\left(b-a_{0}\right) / b$ and $e_{2}=\left(c-a_{0}\right) / c$, where $b$ and $c$ are the substrate lattice parameters and $a_{0}$ is the lattice constant of the cubic paraelectric phase of the film at the stressfree state. The in-plane shear strain is approximately treated to be zero because of the tetragonal of the substrate and because the plane stress condition is held along the film thickness direction due to the thin thickness of the film.

Using the Gibbs free energy, $G,{ }^{6}$ and the Legendre transformation of $F=G+e_{1} \sigma_{1}+e_{2} \sigma_{2}$, the Helmholtz free energy, $F$, of a single domain ferroelectric film under non-equally biaxial inplane misfit strains takes the form:

$$
\begin{aligned}
F & =\alpha_{1}^{*} P_{1}^{2}+\alpha_{2}^{*} P_{2}^{2}+\alpha_{3}^{*} P_{3}^{2}+\alpha_{11}^{*}\left(P_{1}^{4}+P_{2}^{4}\right)+\alpha_{33}^{*} P_{3}^{4}+\alpha_{12}^{*} P_{1}^{2} P_{2}^{2}+\alpha_{13}^{*}\left(P_{2}^{2} P_{3}^{2}+P_{1}^{2} P_{3}^{2}\right) \\
& +\alpha_{111}\left(P_{1}^{6}+P_{2}^{6}+P_{3}^{6}\right)+\alpha_{112}\left[\left(P_{1}^{4}\left(P_{2}^{2}+P_{3}^{2}\right)+P_{2}^{4}\left(P_{1}^{2}+P_{3}^{2}\right)+P_{3}^{4}\left(P_{1}^{2}+P_{2}^{2}\right)\right]\right. \\
& +\alpha_{123} P_{1}^{2} P_{2}^{2} P_{3}^{2}+\frac{s_{11}\left(e_{1}^{2}+e_{2}^{2}\right)-2 s_{12} e_{1} e_{2}}{2\left(s_{11}^{2}-s_{12}^{2}\right)}
\end{aligned}
$$

where the renormalized coefficients $\alpha_{i j}^{*}$ can be found in Ref. 6 and $\alpha_{i}^{*}$ are given by

$$
\begin{gathered}
\alpha_{1}^{*}=\alpha_{1}-\frac{Q_{12}\left(s_{11} e_{2}-s_{12} e_{1}\right)+Q_{11}\left(s_{11} e_{1}-s_{12} e_{2}\right)}{s_{11}^{2}-s_{12}^{2}}, \\
\alpha_{2}^{*}=\alpha_{1}-\frac{Q_{12}\left(s_{11} e_{1}-s_{12} e_{2}\right)+Q_{11}\left(s_{11} e_{2}-s_{12} e_{1}\right)}{s_{11}^{2}-s_{12}^{2}}, \\
\alpha_{3}^{*}=\alpha_{1}-\frac{Q_{12}\left(e_{1}+e_{2}\right)}{s_{11}+s_{12}}
\end{gathered}
$$

In Eqs. (1) and (2), $\alpha_{1}$ is the dielectric stiffness, $\alpha_{i j}$ and $\alpha_{i j k}$ are higher order stiffness coefficients at constant stress, $Q_{i j}$ are the electrostrictive coefficients, and $s_{i j}$ are the elastic compliances of the film. The same material parameters used in Ref. 6 are used in the present work. 
The equilibrium phases under different misfit strains and temperatures are determined by minimization of the Helmholtz free energy. We introduce the following notation for the different equilibrium phases that may occur in the films: (i) the $a_{1}$ phase, where $P_{1} \neq 0$ and $P_{2}=P_{3}=0$; (ii) the $a_{2}$ phase, where $P_{2} \neq 0$ and $P_{1}=P_{3}=0$; (iii) the $c$ phase, where $P_{3} \neq 0$ and $P_{1}=P_{2}=0$; (iv) the $a_{1} c$ phase, where $P_{3} \neq 0, P_{1} \neq 0$ and $P_{2}=0 ;$ (v) the $a_{2} c$ phase, where $P_{3} \neq 0, P_{2} \neq 0$ and $P_{1}=0$; (vi) the $a_{1} a_{2}$ phase, where $P_{2} \neq 0, P_{1} \neq 0$ and $P_{3}=0$; (vii) the $r$ phase, where $P_{3} \neq 0, P_{1} \neq 0$ and $P_{2} \neq 0$. Figs. 1(a) and 1(b) show the misfit strain-misfit strain phase diagrams for epitaxial BT and PT films at room temperature, respectively. For the BT films, the $r$ phase (monoclinic phase) does not exist in the entire region of misfit strains ranging from -0.004 to 0.008 . The other six phases, i.e., three orthorhombic phases $\left(a_{1} a_{2}, a_{1} c, a_{2} c\right)$ and three tetragonal phases $\left(c, a_{1}, a_{2}\right)$, show up in different regions of misfit strains, as shown in Fig. 1(a). The thermodynamics analysis exhibits that the transitions from the $a_{1} a_{2}$ phase to the $c a_{1}$ or $c a_{2}$ phase, and from the $c a_{1}$ phase to the $c a_{2}$ phase are of the first-order, whereas the transitions between two of other phases are of the second order. The $a_{1}$ and $a_{2}$ tetragonal phases appear only in the regions where the non-equal biaxial misfit strains are tensile in one direction and compressive in another direction. The tetragonal $c$ phase exists when the misfit strains are compressive in both directions, whereas the $a_{1} a_{2}$ in-plane orthorhombic phase only exists when the misfit strains are tensile in both directions. For the PT films, $a_{1} c$ and $a_{2} c$ phases do not exist in the entire region of the misfit strains ranging from -0.02 to 0.02 . The other five phases, i.e., $r$, $a_{1} a_{2}, c, a_{1}$ and $a_{2}$, exist within different regions of misfit strains, as shown in Fig. 1(b). The inplane $a_{1}$ or $a_{2}$ tetragonal phase appears in a region of the misfit strains, where one misfit strain 
is compressive and the other is tensile or compressive. For both of the BT and PT films, the nonequally biaxial misfit strains with a compressive component are necessary for the formation of the $a_{1}$ and $a_{2}$ in-plane tetragonal phases, which are forbidden when the misfit strains are equally biaxial, thereby suggesting that the non-equally biaxial misfit strains must be imposed on the ferroelectric films by their substrates in order to obtain the stable and desired in-plane tetragonal phases.

Figs. 2(a) and 2(b) show two-dimensional "temperature-strain" phase diagrams for the BT and PT films, respectively, with $e_{1}=0.005$. The phase transition from the paraelectric state to a single-domain ferroelectric state occurs at a temperature, $T_{c}=\max \left[T_{1}, T_{2}, T_{3}\right]$, where $T_{1}, T_{2}$ and $T_{3}$ denote the temperatures at which the paraelectric phase transfers into the $a_{1}, a_{2}$ and $c$ phases, respectively. Due to the non-equally biaxial misfit strains, the paraelectric-to-ferroelectric transition temperature $T_{c}\left(e_{2}, e_{1}=0.005\right)$ for both $\mathrm{BT}$ and PT films has a plateau above the $a_{1}$ phase, which is different from the "seagull" shape of the transition temperature versus the equal biaxial misfit strain. ${ }^{6}$ The significant feature induced by the non-equally biaxial misfit strains is the formation of the tetragonal ferroelectric phases, $a_{1}$ and $a_{2}$, which do not exist in the BT and PT films under equally biaxial misfit strains. ${ }^{6}$

The misfit strain-induced phase transition may be accompanied by dielectric anomaly. The dielectric constants, $\varepsilon_{i j}$, at small-signals are identical to the reciprocal dielectric susceptibilities, $\chi_{i j}$, which can be calculated from the second partial derivative of the free energy, i.e., $\chi_{i j}=\partial^{2} F / \partial P_{i} \partial P_{j}$. Here, we plot in Fig. 3(b) the relative dielectric constants versus the misfit 
strain, $e_{2}$, with a constant value of the misfit strain of $e_{1}=0.005$ at temperature $300{ }^{\circ} \mathrm{C}$ for the PT films as an example to demonstrate the behavior of dielectric constants under non-equally biaxial misfit strains. To understand the behavior of the dielectric constants, the corresponding polarizations are plotted in Fig. 3(a). At the phase boundary between the $c$ and $a_{1}$ phases, polarization components $P_{1}$ and $P_{3}$ are discontinuous, thereby indicating the phase transition is of the first order. The discontinuity of the $P_{1}$ and $P_{3}$ polarization components cross the boundary between the $c$ and $a_{1}$ phases causes the anomalies in the $\varepsilon_{11}$ and $\varepsilon_{33}$ dielectric constants and the jump in the $\varepsilon_{22}$ dielectric constant, as shown in Fig. 3(b), because the $\varepsilon_{22}$ dielectric constant is related to the $P_{1}$ and $P_{3}$ polarization components.

In summary, the Helmholtz free energy, $F$, for ferroelectric thin films under non-equally biaxial misfit strains has been explicitly given, from which the relationships among the two misfit strains and temperature, i.e., the three-dimensional phase diagram can be constructed and the dielectric constant can be investigated as a function of temperature and the misfit strains. The present work is based on the single domain assumption, as many thermodynamic approaches do. ${ }^{6-10}$ Neither domain walls nor the long-range mechanical and electrical interactions are taken into account. The $a_{1} a_{2}, a_{1} c, a_{2} c$ and $r$-phases may represent the $a_{1} / a_{2} / a_{1} / a_{2}, a_{1} / c / a_{1} / c$, $a_{2} / c / a_{2} / c$ and $c / a_{1} / a_{2}$ multidomain structures, respectively. In this sense, the thermodynamics results based on the single domain assumption may be regarded as the macroscopic average of multidomain structures. Of course, the phase boundaries predicted by the thermodynamics approaches may be different from those in multidomain ferroelectric thin films. 
This work was fully supported by an RGC grant from the Research Grants Council of the Hong Kong Special Administrative Region, China. 


\section{References}

${ }^{1}$ M. J. Dalberth, R. E. Stauber, J. C. Price, C. T. Roger, and D. Galt, Appl. Phys. Lett. 72, 507 (1998).

${ }^{2}$ Q. X. Jia, A. T. Findikoglu, D. Reagor, and P. Lu, Appl. Phys. Lett. 73, 897 (1998).

${ }^{3}$ H.N. Lee, D. Hesse, N. Zakharov, and U. Gosele, Science 296, 2006 (2002).

${ }^{4}$ C. H. Ahn, K. M. Rabe, and J.-M. Triscone, Science 303, 488 (2004).

${ }^{5}$ C. L. Chen, H. H. Feng, Z. Zhang, A. Brazdeikis, Z. J. Huang, W. K. Chu, C. W. Chu, F. A. Miranda, F. W. Van Keuls, R. R. Romanofsky, and Y. Liou, Appl. Phys. Lett. 75, 412 (1999).

${ }^{6}$ N.A. Pertsev, A.G. Zembilgotov, and A.K. Tagantsev, Phys. Rev. Lett. 80, 1988 (1998).

${ }^{7}$ B.S. Kwak, A. Erbil, B.J. Wilkens, J.D. Budai, M.F. Chisholm, and L.A. Boatner, Phys. Rev. Lett. 68 , 3733 (1992).

${ }^{8}$ S.H. Oh, and H.M. Jang, Appl. Phys. Lett. 72, 1457 (1998).

${ }^{9}$ A.Y. Emelyanov, N.A. Pertsev, and A.L. Kholkin, Phys. Rev. B 66, 214108 (2002).

${ }^{10}$ S.H. Oh, and H.M. Jang, Phys. Rev. B 62, 14757 (2000).

${ }^{11}$ A. Amin, R.E. Newnhan, and L.E. Cross, Phys. Rev. B 34, 1595 (1986).

${ }^{12}$ V.G. Koukhar, N.A. Pertsev, and R. Waser, Appl. Phys. Lett. 78, 530 (2001).

${ }^{13}$ Y.L. Li, S.Y. Hu, Z.K. Liu, and L.Q. Chen, Appl. Phys. Lett. 78, 3878 (2001).

${ }^{14}$ Z.G. Ban, and S.P. Alpay, J. Appl. Phys. 91, 9288 (2002).

${ }^{15}$ Z.G. Ban, and S.P. Alpay, J. Appl. Phys. 93, 504 (2003).

${ }^{16}$ A.L. Roytburd, J. Appl. Phys. 83, 228 (1998).

${ }^{17}$ Y. Lin, X. Chen, S.W. Liu, C.L. Chen, J.S. Lee, Y. Li, Q.X. Jia and A. Bhalla, Appl. Phys. Lett. 84, 577 (2004).

${ }^{18}$ H.N. Lee, and D. Hesse, Appl. Phys. Lett. 80, 1040 (2002).

${ }^{19}$ A. Garga, Z. H. Barber, M. Dawber, J. F. Scott, A. Snedden, and P. Lightfoot, Appl. Phys. Lett. 83, $2414(2003)$. 


\section{Figure captions}

FIG.1. Misfit strain - misfit strain phase diagrams of single-domain BT (a) and PT (b) thin films at room temperature, where the thin and thick lines denote the second- and first-order phase transition boundaries, respectively

FIG.2. Temperature - misfit strain phase diagrams, at a constant misfit strain of $e_{1}=0.005$, of single-domain BT (a) and PT (b) thin films, where the thin and thick lines denote the secondand first-order phase transition boundaries, respectively.

FIG.3. Dependence of polarizations (a) and relative dielectric constants (b) on the misfit strain, $e_{2}$, in single-domain PT films with a constant misfit strain of $e_{1}=0.005$ at $300^{\circ} \mathrm{C}$. 

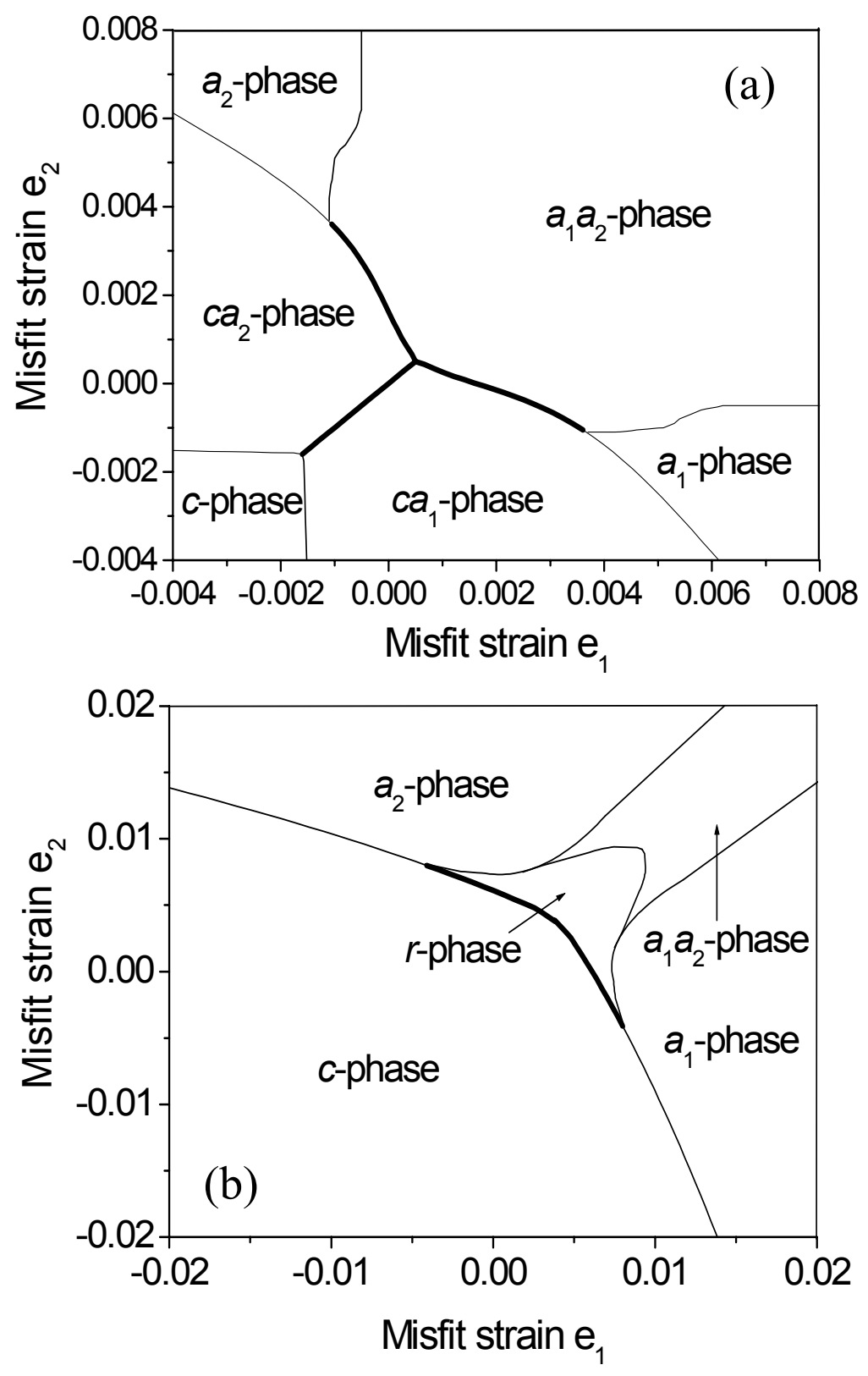

FIG.1. 

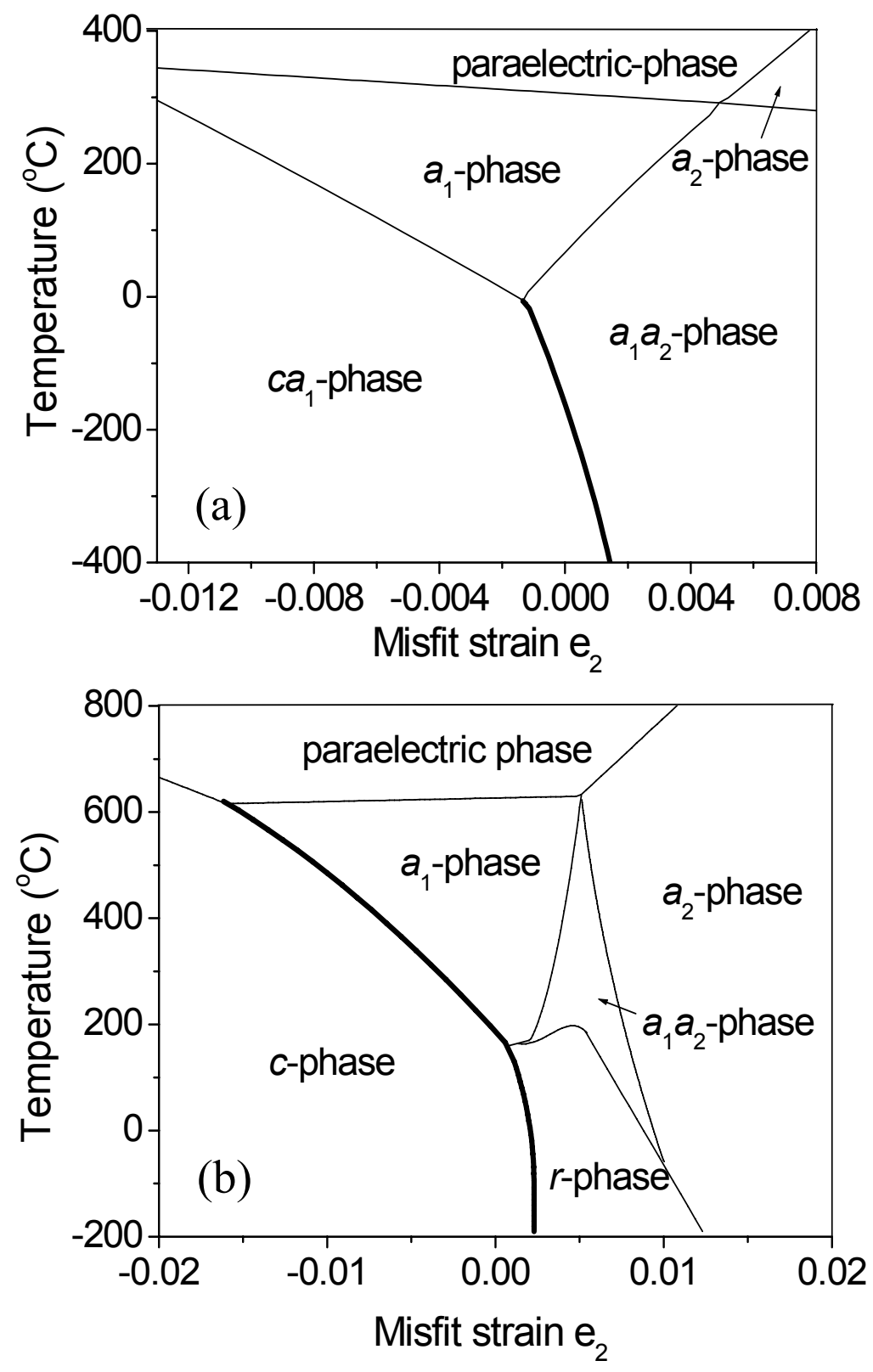

FIG.2. 

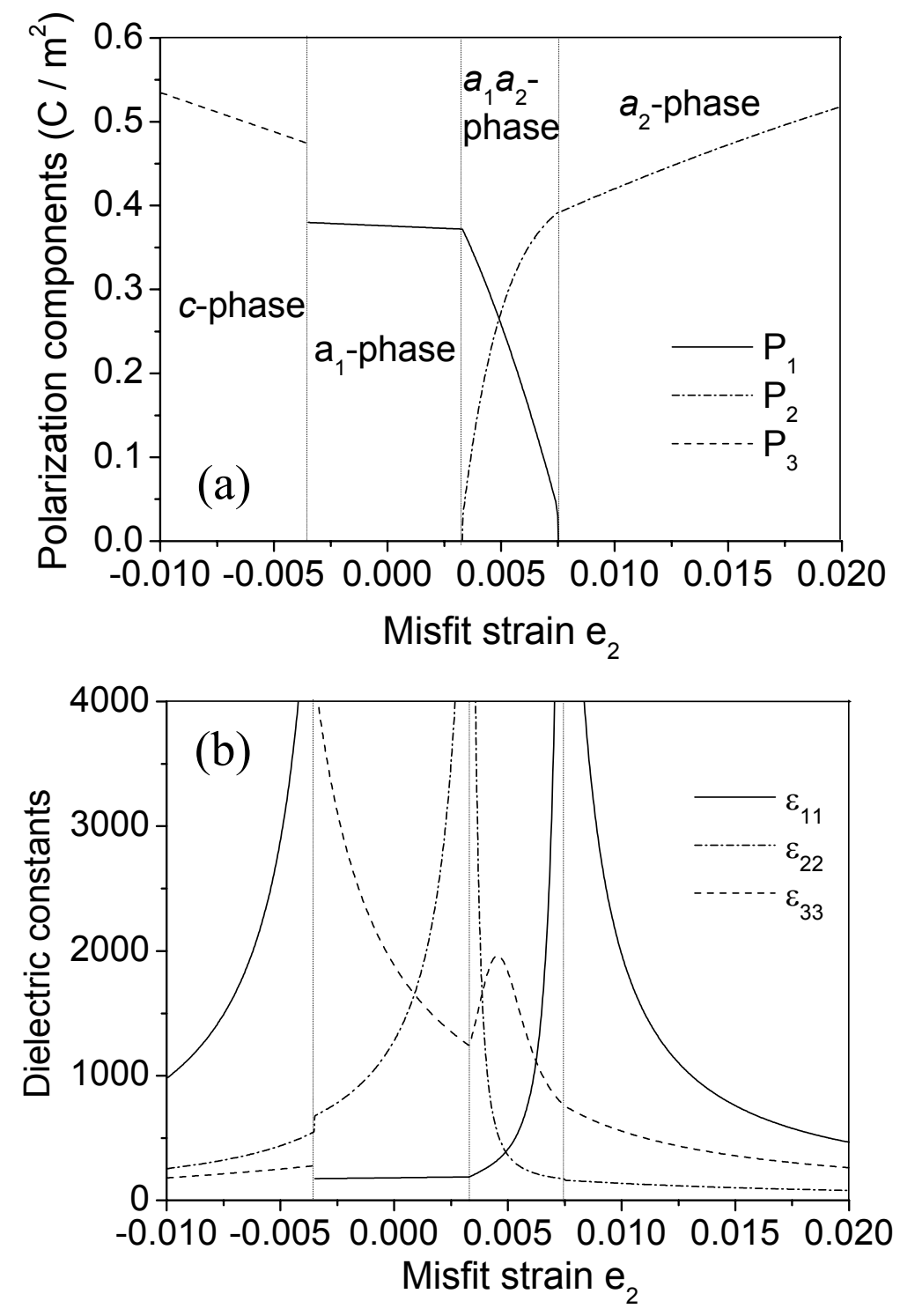

FIG.3. 